\title{
Vascular endothelial syndromes after HCT: 2020 update
}

\author{
Enric Carreras $\mathbb{D}^{1,2} \cdot$ on behalf of the Barcelona Endothelium Team (BET)
}

Received: 26 December 2019 / Revised: 2 January 2020 / Accepted: 26 February 2020 / Published online: 12 March 2020

(c) Springer Nature Limited 2020

Given that it is several years since the publication in Bone Marrow Transplantation of our first editorial focusing on the endothelial complications shortly after hematopoietic cell transplantation (HCT) [1], we felt it is of utmost importance to update the topic. Several advances have been made since that publication to justify such an update and a recent review on the subject by Pagliuca et al. [2], suggested that an update is now timely. The current contribution aims to offer a thorough review of the current knowledge of the main vascular endothelial syndromes (VES) and at the same time introduces some new concepts and suggests several changes to the classification that we think deserve in-depth comment.

The first aspect of the above mentioned review article [2] that may lead to confusion is the title "Allogeneic reactivity-mediated endothelial cell complications," since all VES, except the recently introduced graft-versus-host disease (GVHD) (see below), can also be observed after autologous HCT. Allogeneic reactivity is only one of the factors that induces endothelial dysfunction. In fact, one of these complications, the engraftment syndrome [3], is almost exclusively seen after auto-HCT; others, such as diffuse alveolar hemorrhage (DAH) or peri-engraftment respiratory distress syndrome (PERDS), may be observed in both modalities of transplantation $[4,5]$, and transplantassociated thrombotic microangiopathy (TA-TMA) despite being observed mainly after allo-HCT, can occasionally be diagnosed after auto-HCT [6]. An excellent example of the impact of the alloreactivity in VES is offered by sinusoidal

Members of the Barcelona Endothelium Team are listed below funding.

Enric Carreras

enric@fcarreras.es

Josep Carreras Leukemia Foundation, Barcelona, Spain

2 Josep Carreras Leukemia Research Institute, Hospital Clinic/UB Campus, Barcelona, Spain obstruction syndrome (SOS) as its incidence correlates perfectly with the intensity of alloreactivity. Thus, a higher incidence of SOS will occur after allogeneic (versus autologous), unrelated (versus sibling), mismatched (versus matched) or unmanipulated (versus T-cell depleted) HCT [7]. Consequently, sensu strictu, we should not speak of VES mediated by alloreactivity but of the impact of alloreactivity on VES.

The second questionable aspect of the review article [2] is the proposal to remove SOS from VES list because "it's pathogenesis is not entirely endothelial." The question immediately arises: is there any evidence that the remaining VES are entirely endothelial? If we analyze, for example, one of the most important VES, that of TA-TMA, we can observe that in addition to the initial endothelial dysfunction, many other pathogenetic events occur: thrombus formation in microcirculation (origin of the classic manifestations of this disease); activation of antigen-presenting cells (with the possible production of antibodies against factor $\mathrm{H}$ and recipient-specific antibodies); overproduction of neutrophil extracellular traps that facilitate (together with some gene polymorphisms) the activation of the classical and alternative complement pathway with deposition of C4d and C5b-9 or membrane attack complex in the renal arterioles [6, 8]. Therefore, TA-TMA is also not an entirely endothelial complication. A final argument against the exclusion of SOS from the VES is to recognize the historical fact that after knowing the endothelial origin of SOS [9] and observing that other early complications of HCT had a close relationship with the microvascular tree and overlapping clinical manifestations, all these syndromes were grouped under the designation of VES [10]. Therefore, the exclusion of SOS would render the classical VES classification meaningless.

Despite it being usual to include idiopathic pneumonia syndromes (IPS) among the VES, this isn't entirely correct. Most IPS do not have a well-known/understood pathogenesis but the fact that cannot be denied is that only a small subgroup of IPS affect the vascular tree, including DAH, 
Table 1 Vascular endothelial syndromes after HCT.

Sinusoidal obstruction syndrome (SOS)

Capillary leak syndrome (CLS)

Pre-engraftment syndrome (pre-ES)

Engraftment syndrome (ES)

Transplant-associated thrombotic microangiopathy (TA-TMA)

Vascular idiopathic pneumonia syndromes:

Diffuse alveolar hemorrhage (DAH)

Capillary leak syndrome (CLS)

Peri-engraftment respiratory distress syndrome (PERDS)

Posterior reversible encephalopathy syndrome (PRES)

Acute-graft-versus-host disease (aGVHD) (provisional entity)

PERDS, and capillary leak syndrome (CLS) [5]. Consequently, the correct way of classifying these VES that affect the lungs is with the designation "Vascular IPS".

A common origin shared between VES and acute GVHD had already been proposed several years ago. Initial observations suggested the hypothesis of vascular endothelium as a target of GVHD [11]. Nowadays, there is enough evidence indicating that endothelial damage is not only a consequence of GVHD but the possible triggering event of this complication [12]. In addition, it has been observed that angiogenesis observed in GVHD occurs exclusively in target organs and precedes leukocyte infiltration during GVHD [13]. Despite all these relationships, it is difficult to accept the sentence "VES may overlap with acute GVHD and, sometimes, the clinical distinction among these forms and GVHD can be challenging" as the clinical manifestations of GVHD differ completely, even in the hyperacute forms [14], from those observed in VES, except in some cases of intestinal TA-TMA and GVHD [15]. In fact, this observation is one of the main reasons for reluctancy to include GVHD among VES, since its clinical behavior does not overlap with those of the rest of VES.

The last aspect to comment on in a manuscript dedicated to VES and its treatment is the absence of some detailed references on the protective and therapeutic effect of defibrotide on the endothelium. This agent has demonstrated its effectiveness in: treatment of severe SOS [16]; prevention of SOS and GVHD in a randomized study in children [17] (basis of the current randomized study of GVHD prevention with defibrotide ClinicalTrials.gov-NCT03339297); treatment of TA-TMA [18]; prevention and treatment of GVHD in vitro [19] and in animal models (manuscript submitted). If this effect is confirmed in the ongoing studies, we will have the first agent preventing GVHD without causing immunosuppression.

In conclusion, we propose only minor changes to the original classification of VES (see Table 1).
Funding This work was partially supported by Deutsch José Carreras Leukämie Stiftung (grant 11R/2016 and 03R/2019) and Jazz Pharmaceuticals (IST-16-10355).

Barcelona Endothelium Team (BET) Marta Palomo², Maribel Diaz Ricart $^{3}$, Julia Martínez-Sánchez ${ }^{2}$

${ }^{3}$ Department of Hematopathology, Biomedical Diagnosis Center (CDB), Institute of Biomedical Research August Pi i Sunyer (IDIBAPS), Hospital Clinic of Barcelona, University of Barcelona, Barcelona, Spain

\section{Compliance with ethical standards}

Conflict of interest BET activity is supported in part by a grant from Jazz Pharmaceutical plc/Gentium Inc. EC forms part of the speaker's bureau of Jazz Pharmaceutical plc.

Publisher's note Springer Nature remains neutral with regard to jurisdictional claims in published maps and institutional affiliations.

\section{References}

1. Carreras E, Diaz-Ricart M. The role of the endothelium in the short-term complications of hematopoietic SCT. Bone Marrow Transpl. 2011;46:1495-502.

2. Pagliuca S, Michonneau D, Sicre de Fontbrune F, Sutra Del Galy A, Xhaard A, Robin M, et al. Allogeneic reactivity-mediated endothelial cell complications after HSCT: a plea for consensual definitions. Blood Adv. 2019;3:2424-35.

3. Carreras E, Fernández-Avilés F, Silva L, Guerrero M, Fernández de Larrea C, Martínez C, et al. Engraftment syndrome after auto-SCT: analysis of diagnostic criteria and risk factors in a large series from a single center. Bone Marrow Transpl. 2010;45:1417-22.

4. Afessa B, Tefferi A, Litzow MR, Peters SG. Outcome of diffuse alveolar hemorrhage in hematopoietic stem cell transplant recipients. Am J Respir Crit Care Med. 2002;166:1364-8.

5. Panoskaltsis-Mortari A, Griese M, Madtes DK, Belperio JA, Haddad IY, Folz RJ, Cooke KR, American Thoracic Society Committee on Idiopathic Pneumonia Syndrome. An official American Thoracic Society research statement: noninfectious lung injury after hematopoietic stem cell transplantation: idiopathic pneumonia syndrome. Am J Respir Crit Care Med. 2011;183:1262-79.

6. Jodele S, Laskin BL, Dandoy CE, Myers KC, El-Bietar J, Davies SM, et al. A new paradigm: diagnosis and management of HSCT-associated thrombotic microangiopathy as multi-system endothelial injury. Blood Rev. 2015;29:191-204.

7. Carreras E. How I manage sinusoidal obstruction syndrome after haematopoietic cell transplantation. Br J Haematol. 2015; 168:481-91.

8. Gloude NJ, Khandelwal P, Luebbering N, Lounder DT, Jodele S, Alder MN, et al. Circulating dsDNA, endothelial injury, and complement activation in thrombotic microangiopathy and GVHD. Blood. 2017;130:1259-66.

9. DeLeve LD, McCuskey RS, Wang X, Hu L, McCuskey MK, Epstein RB, Kanel GC. Characterization of a reproducible rat model of hepatic veno-occlusive disease. Hepatology. 1999;29:1779-91.

10. Carreras E. Early complications after HSCT. In: Apperley J, Carreras E, Gluckman E, Gratwohl A, Masszi T, editors. The ebmt 
handbook: haemopoietic stem cell transplantation. Switzerland: Forum Service Editore; 2004. p. 133-45.

11. Tichelli A, Gratwohl A. Vascular endothelium as 'novel' target of graft-versus-host disease. Best Pract Res Clin Haematol. 2008; 21:139-48.

12. Penack O, Socié G, van den Brink MR. The importance of neovascularization and its inhibition for allogeneic hematopoietic stem cell transplantation. Blood. 2011;117:4181-9.

13. Riesner K, Shi Y, Jacobi A, Kräter M, Kalupa M, McGearey A, et al. Initiation of acute graft-versus-host disease by angiogenesis. Blood. 2017;129:2021-32.

14. Saliba RM, de Lima M, Giralt S, Andersson B, Khouri IF, Hosing C, et al. Hyperacute GVHD: risk factors, outcomes, and clinical implications. Blood. 2007;109:2751-8.

15. Gavriilaki E, Sakellari I, Karafoulidou I, Pasteli N, Batsis I, Mallouri D, et al. Intestinal thrombotic microangiopathy: a distinct entity in the spectrum of graft-versus-host disease. Int J Hematol. 2019;110:529-32.
16. Richardson PG, Carreras E, Iacobelli M, Nejadnik B. The use of defibrotide in blood and marrow transplantation. Blood Adv. 2018;2:1495-509.

17. Corbacioglu S, Cesaro S, Faraci M, Valteau-Couanet D, Gruhn B, Rovelli A, et al. Defibrotide for prophylaxis of hepatic venoocclusive disease in paediatric haemopoietic stem-cell transplantation: an open-label, phase 3, randomised controlled trial. Lancet. 2012;379:1301-9.

18. Yeates L, Slatter MA, Bonanomi S, Lim FLWI, Ong SY, Dalissier A, et al. Use of defibrotide to treat transplantassociated thrombotic microangiopathy: a retrospective study of the paediatric diseases and inborn errors working parties of the european society of blood and marrow transplantation. Bone Marrow Transpl. 2017;52:762-4.

19. Martinez-Sanchez J, Hamelmann H, Palomo M, Mir E, MorenoCastaño AB, Torramade S, et al. Acute graft-vs.-host diseaseassociated endothelial activation in vitro is prevented by defibrotide. Front Immunol. 2019;10:2339. 